Instructors

Journalists

Librarians

Rights \& Permissions

Societies \& Publishing Partners

Subscription Agencies

Help \& Contact

Open Access \& Springer

Products

Journals

Books

Proceedings

SpringerLink

Springer for R\&D

Springer for Hospitals \& Health

Databases and Software

Springer Shop

About us

$50 \%$ off Statistics books + FREE shipping +++ All Engineering eBooks \& journals 50\% off

Business \& Management Operations Research \& Decision Theory

Free Preview

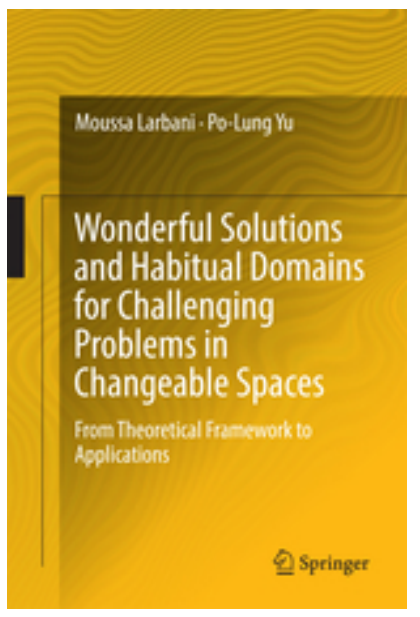

(C) 2017

\title{
Wonderful Solutions and Habitual Domains for Challenging Problems in Changeable Spaces
}

\section{From Theoretical Framework to Applications}

Authors: Larbani, Moussa, Yu, Po-Lung

Introduces a new decision theory, Optimization in Changeable Spaces

Illustrates how to expand competences, handle environmental, psychological and behavioral aspects and their dynamics when solving challenging decision problems

Includes perspectives related to innovation dynamics, management, artificial intelligence, artificial and e-economics, scientific discovery and knowledge extraction

Relevant to academics, managers and policy makers

see more benefits

Buy this book

eBook

$118,99 €$

price for Malaysia (gross) 
Buy eBook

ISBN 978-981-10-1981-4

Digitally watermarked, DRM-free

Included format: PDF, EPUB

ebooks can be used on all reading devices

Immediate eBook download after purchase

Hardcover

$149,99 €$

price for Malaysia (gross)

Buy Hardcover

ISBN 978-981-10-1979-1

Free shipping for individuals worldwide

Usually dispatched within 3 to 5 business days.

FAQ Policy

About this book

About the authors

Reviews

About this book

This book introduces a new paradigm called 'Optimization in Changeable Spaces' (OCS) as a useful tool for decision making and problem solving. It illustrates how OCS incorporates, searches, and constructively restructures the parameters, tangible and intangible, involved in the process of decision making. The book elaborates on OCS problems that can be modeled and solved effectively by using the concepts of competence set analysis, Habitual Domain (HD) and the mental operators called the 7-8-9 principles of deep knowledge of HD. In addition, new concepts of covering and discovering processes are proposed and formulated as mathematical tools to solve OCS problems. The book also includes reformulations of a number of illustrative real-life challenging problems that cannot be solved by traditional optimization techniques into OCS problems, and details how they can be addressed. Beyond that, it also includes perspectives related to innovation dynamics, management, artificial intelligence, artificial and e-economics, scientific discovery and knowledge extraction. This book will be of interest to managers of businesses and institutions, policy makers, and educators and students of decision making and behavior in DBA and/or MBA.

Show all

About the authors

Reviews

\section{Table of contents}

\section{(10 chapters)}

Challenging Decision Problems and Decision Models

Larbani, Moussa (et al.)

Pages 1-23

Preview Buy Chapter

\section{$24,95 €$}

Decision Processes and Decision-Making in Changeable Spaces

Larbani, Moussa (et al.)

Pages 25-50

Preview Buy Chapter

\section{$24,95 €$}

Habitual Domains, Human Behaviour Mechanism and Wonderful Solutions for DMCS Problem Analysis

Larbani, Moussa (et al.)

Pages 51-88

Preview Buy Chapter

$24,95 €$

Expansion of Habitual Domains and DMCS

Larbani, Moussa (et al.)

Pages 89-121

Preview Buy Chapter

\section{$24,95 €$}


Competence Set Analysis, Decision Blinds and Decision-Making

Larbani, Moussa (et al.)

Pages 123-145

Preview Buy Chapter

$24,95 €$

Show next

5

Read this book on SpringerLink

Download Preface 1 PDF

(133.3 KB)

Download Sample pages 2 PDF

(382.4 KB)

Download Table of contents PDF

(135.7 KB)

Buy this book

eBook

$118,99 €$

price for Malaysia (gross)

Buy eBook

ISBN 978-981-10-1981-4

Digitally watermarked, DRM-free

Included format: PDF, EPUB

ebooks can be used on all reading devices

Immediate eBook download after purchase

Hardcover

$149,99 €$

price for Malaysia (gross)

Buy Hardcover

ISBN 978-981-10-1979-1

Free shipping for individuals worldwide

Usually dispatched within 3 to 5 business days.

FAQ Policy

\section{Book Metrics}

Reviews

1

Downloads

1156

Provided by Bookmetrix

\section{Services for this Book}

Download Product Flyer Reserve an Online Book Review Copy Download High-Resolution Cover

Facebook

Twitter

Linkedln

Google++

\section{Recommended for you}




\section{Contents}

1 Challenging Decision Problems and Decision Models ........... 1

$1.1 \quad$ Introduction . . . . . . . . . . . . . . . . . . . . . . 1

1.2 Existing Decision-Making Models and Their Limitations ....... . 2

1.2.1 Traditional Decision Models ................. 2

1.2.2 The Cognitive Decision Models............... 6

1.2.3 Some Problems Unsolvable by Existing

Decision Models. ........................... 6

1.3 An Informal Introduction to Decision-Making

in Changeable Spaces ......................... 11

1.4 Conclusion................................. 13

Appendix 1: A Snapshot on Decision-Making Models............ 14

Appendix 2: Discussion of Schelling's Game of Chicken

Model of Problem 1.8 (Cuban Missile Crisis) . . . . . . . . 20

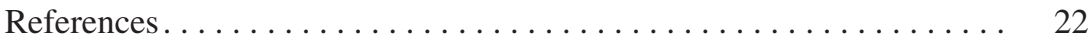

2 Decision Processes and Decision-Making in Changeable Spaces .... 25

2.1 Introduction ............................ 25

2.1.1 A Brief Introduction to the Ten Decision Parameters ..... 26

2.2 Classification of Decision Problems ............... 30

2.3 Decision Elements and Decision Environmental Facets ........ 32

2.3.1 Decision Elements . . . . . . . . . . . . . . . . 32

2.3.2 Decision Environmental Facets . . . . . . . . . . . 38

2.4 Decision-Making in Changeable Spaces Problems............. 44

2.5 Conclusion................................ 49

References .............................. 50

3 Habitual Domains, Human Behaviour Mechanism

and Wonderful Solutions for DMCS Problem Analysis ......... 51

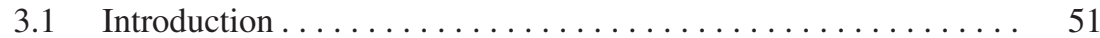

3.2 Habitual Domains .......................... 52

3.3 The Eight HD Hypotheses H1-H8 in Behaviour Dynamics..... 54

3.3.1 Circuit Pattern Hypothesis, H1 . . . . . . . . . . . . . . 54 
3.3.2 Unlimited Capacity Hypothesis, H2 . . . . . . . . . . 55

3.3.3 Efficient Restructuring Hypothesis, $\mathrm{H} 3 \ldots \ldots \ldots \ldots \ldots . \quad 55$

3.3.4 Analogy/Association Hypothesis, $\mathrm{H} 4 \ldots \ldots \ldots \ldots \ldots .66$

3.3.5 Goal Setting and State Evaluation Hypothesis, H5 . . . . . 57

3.3.6 Charge Structure and Attention Allocation Hypothesis, H6 . . . . . . . . . . . . . . . . . . . . . 60

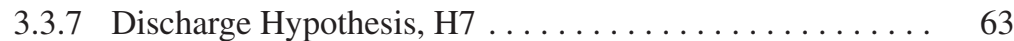

3.3.8 Information Input Hypothesis, $\mathrm{H} 8 \ldots \ldots \ldots \ldots \ldots \ldots \ldots$

3.4 Human Behaviour Mechanism and Decision-Making ........ 66

3.4.1 Stability of Habitual Domains .................. 69

3.5 HD Model of DMCS Problems and Acceptable and Wonderful Solutions ....................... 71

3.5.1 Acceptable Solutions and Wonderful Solutions.......... 74

3.5.2 Comparing DMCS Habitual Domain Model with Existing Decision Models ................. 78

3.6 Behavioural Tendencies.......................... 81

3.7 Conclusion................................ 87

References .............................. 88

4 Expansion of Habitual Domains and DMCS. . . . . . . . . . . . 89

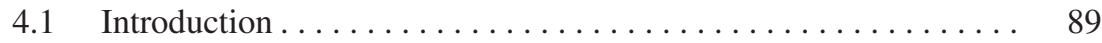

4.2 Degrees of HD Expansion..................... 91

4.2.1 Zero-Degree Expansion ..................... 91

4.2.2 First-Degree Expansion ........................ 92

4.2 .3 Second-Degree Expansion ................... 93

4.3 The 7-8-9 Principles of Deep Knowledge for HD Expansion..... 95

4.3.1 Seven Empowerment Operators.................. 96

4.3.2 Eight Basic Methods for Expanding HD . . . . . . . . . . 101

4.3.3 Nine Principles for Deep Knowledge. . . . . . . . . . . . . 105

4.4 Procedure for Solving DMCS Problems . . . . . . . . . . . . . . . 112

4.5 Conclusion............................. 120

References ............................. 120

5 Competence Set Analysis, Decision Blinds and Decision-Making. . . . 123

5.1 Introduction ............................ 123

5.2 Cores of Habitual Domains . . . . . . . . . . . . . . . 126

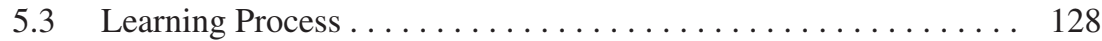

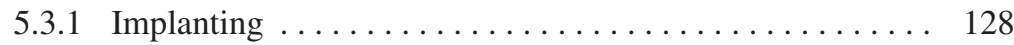

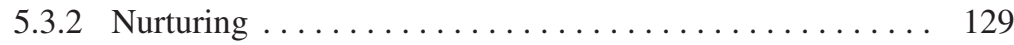

5.3 .3 Habituating....................... 130

5.4 Competence Sets and Classes of Decision Problems ......... 130

5.4 .1 Routine Problem. . . . . . . . . . . . . . . . . . . . 131

5.4.2 Mixed Routine Problems . . . . . . . . . . . . . . . . . . 132

5.4 .3 Fuzzy Problems .......................... 132

5.4.4 Challenging Problems $\ldots \ldots \ldots \ldots \ldots \ldots \ldots \ldots \ldots \ldots \ldots$

5.5 Confidence, Risk Taking and Ignorance . . . . . . . . . . . 136 
5.6 Effective Decision-Making . . . . . . . . . . . . . . 139

5.7 Decision Blinds and Decision Traps . . . . . . . . . . . . . 140

5.8 Covering and Discovering Problems . . . . . . . . . . . . . . 141

5.9 Support in Decision-Making Process . . . . . . . . . . . . . . . . . . 144

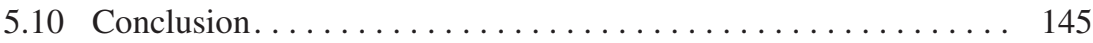

References................................ 145

6 Decision-Making in Changeable Spaces (DMCS):

A New Paradigm . . . . . . . . . . . . . . . . . . . . . 147

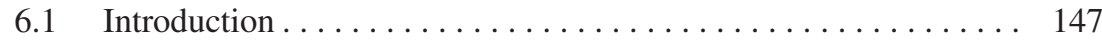

6.2 Optimisation in Changeable Spaces . . . . . . . . . . . . . . . . . . 148

6.3 Covering Problem . . . . . . . . . . . . . . . . . . . . 152

6.3.1 Feasibility and Covering Time and/or Cost . . . . . . . . 154

6.4 Discovering ......................... 155

6.5 Necessary and Sufficient Conditions for Covering . . . . . . . . . . 158

6.5 .1 Cardinality Approach to Covering . . . . . . . . . . . 158

6.6 General Procedures for Solving Covering and Discovering Problems................... 161

6.6.1 General Covering Problem Procedure . . . . . . . . . . . . 164

6.6 .2 Covering Feasibility Procedure . . . . . . . . . . . . 171

6.6 .3 Covering Time Procedure. . . . . . . . . . . . . . . 172

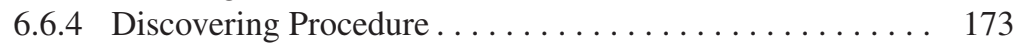

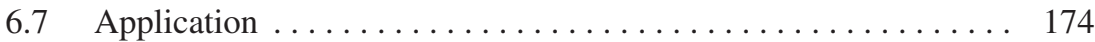

6.8 A Comparison Between OCS Models and Existing

Decision Models . . . . . . . . . . . . . . . . . . . . . . . . . . 180

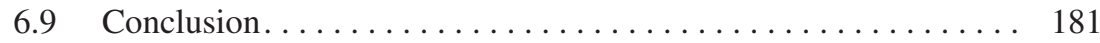

References ................................ 182

\section{Solving Real-World DMCS Problems, Part 1: Management}

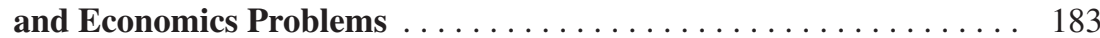

7.1 Introduction . . . . . . . . . . . . . . . . . . . . . . 183

7.2 Management Applications. . . . . . . . . . . . . . . . . . . . . . 184

7.2.1 From Business Crisis to Prosperity by Matsushita. . . . . . . 185

7.2.2 The 1984 Olympic Games, Converting Potential

Big Loss to Big Gains . . . . . . . . . . . . . . . . . 191

7.2.3 From Tough Competition to Supply Chain Integration by Synnex . . . . . . . . . . . . . . . . . . . . . . . 195

7.2.4 Converting Revenge Sentiment to Full Cooperation ... . . . 199

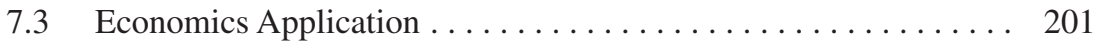

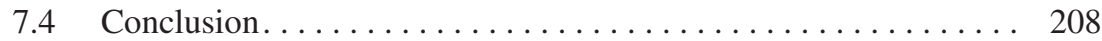

References......................... 208

8 Solving Real-World DMCS Problems, Part 2: Social,

Geopolitical, and Discovery Problems . . . . . . . . . . . . . . . . . . . . 209

8.1 Introduction . . . . . . . . . . . . . . . . . . . . . . . . . . 209

8.2 Silence Game Between Husband and Wife. . . . . . . . . . . . . . 210 
8.3 Clearing a Violent Demonstration Peacefully ............ 214

8.4 The Farmer and the Hunter . . . . . . . . . . . . . . . . 217

8.5 A Winning Strategy Without Implementation . . . . . . . . . . . . . 221

8.6 Cuban Missile Crisis (1962) . . . . . . . . . . . . . . . . . . . . . . 224

8.7 Multilanguage Script Keyboard. . . . . . . . . . . . . . 235

8.8 Conclusion............................. 238

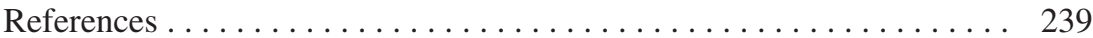

9 Innovation Dynamics as a DMCS Problem . . . . . . . . . . 241

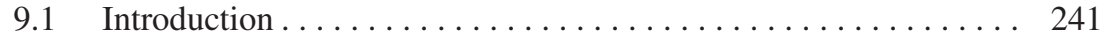

9.2 Innovation from Habitual Domain Perspective . . . . . . . . . . . . 242

9.3 An Anatomy of Innovation Dynamics. . . . . . . . . . . . . . . 243

9.3.1 Competence Set Expansion and Transformation (see Fig. 9.1(i) and (C)) . . . . . . . . . . . . . . . . 244

9.3.2 Providing Product/Service to Release the Pain and Frustration of Targeted Customers

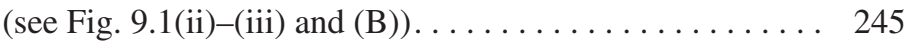

9.3.3 Creating Charge and Releasing Charge

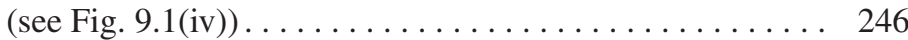

9.3.4 Creating Value (see Fig. 9.1(v) and (A)) .......... 247

9.3.5 Value Distribution and Reinvestment (see Fig. 9.1(vi)

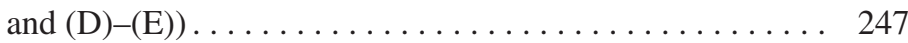

9.3.6 Clockwise and Counterclockwise Versions of Innovation Dynamics . . . . . . . . . . . . . . . 248

9.4 DMCS Problems in Innovation Dynamics . . . . . . . . . . . . . . . 249

9.4.1 OCS and Innovation Dynamics .............. 251

9.5 Conclusion................................ 254

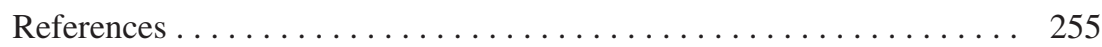

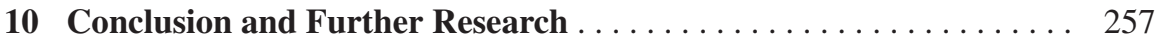

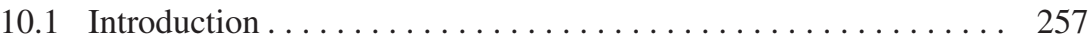

10.2 DMCS and OCS in Management and Game Situations . . . . . . . 258

10.3 DMCS and OCS in Artificial Intelligence. . . . . . . . . . . 259

10.3.1 HD-Agent in Artificial Economics and e-Economy. . . . . . 260

10.4 Scientific Discovery. . . . . . . . . . . . . . . . . . . 261

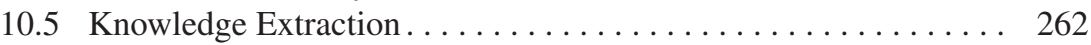

10.6 Competence Set-Related Research Problems . . . . . . . . . . . . 262

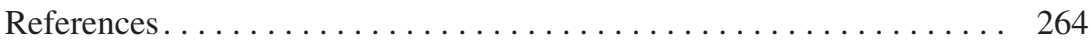

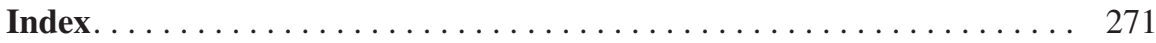




\section{Springer}

http://www.springer.com/978-981-10-1979-1

Wonderful Solutions and Habitual Domains for Challenging Problems in Changeable Spaces From Theoretical Framework to Applications Larbani, M.i Yu, P. -L. 2017, XXVll, 275 p. 17 illus., Hardcover ISBN: 978-981-10-1979-1 